\title{
(n-2)-TIGHTNESS AND CURVATURE OF SUBMANIFOLDS WITH BOUNDARY
}

\author{
WOLFGANG KÜHNEL \\ Fachbereich Mathematik \\ Technische Universität Berlin \\ Straße des 17 . Juni 135 \\ 1000 Rerlin 12 (West) \\ Germany
}

(Received August 2, 1978)

ABSTRACT. The purpose of this note is to establish a connection between the notion of (n-2)-tightness in the sense of N.H. Kuiper and T.F. Banchoff and the total absolute curvature of compact submanifolds-with-boundary of even dimension in Euclidean space. The argument used is a certain geometric inequality similar to that of S.S. Chern and R.K. Lashof where equality characterizes (n-2)-tightness.

KEY WORDS AND PHRASES. tight manifolds, total absolute curvature. AMS (MOS) SUBJECT CLASSIFICATION (1970) CODES. Primary 53 C 40, 57040. 


\section{W. KÜHNEL}

\section{INTRODUCTION.}

Let $M$ be a compact $n$-dimensional smooth manifold with or without boundary where the boundary is assumed to be smooth - and let

$$
\mathrm{f}: \mathrm{M} \longrightarrow \mathrm{E}^{\mathrm{n}+\mathrm{k}}
$$

be a smooth immersion of $M$ into the ( $n+k)$-dimensional euclidean space. This leads to the notion of total absolute curvature

$$
T A(f)=\frac{1}{c_{n+k-1}} \int_{N}|K| \star 1
$$

where $K$ denotes the Lipschitz-Killing curvature of $f$ in each normal direction, $\mathrm{N}$ the unit normal bundle (with only the 'outer' normals at points of $\partial \mathrm{M}$ ), and $\mathrm{c}_{\mathrm{m}}$ denotes the volume of the unit sphere $s^{\mathrm{m}} \subseteq \mathrm{E}^{\mathrm{m}+1}$. For detailed definitions, in particular in the case of manifolds with boundary, see[5] or [6]. Let us state the following equation ( $[6], 2.2)$

$$
T A(f)=T A\left(\left.f\right|_{M \backslash \partial M}\right)+\frac{1}{2} T A(f \mid \partial M)
$$

The famous result of S.S. Chern and R.F. Lashof gives a connection between total absolute curvature and the number of critical points of so-called height functions

$$
\mathrm{zf}: \mathrm{M} \longrightarrow \mathbb{R}
$$

defined by $(z f)(p)=\left\langle z, f(p)>\quad, \quad z \in S^{n+k-1}\right.$

Extending this result to the case of manifolds with boundary we can write

$$
T A(f)=\frac{1}{c_{n+k-1}} \quad z \in S^{n+k-1} \sum_{i}\left(\mu_{i}(z f)+\mu_{i}^{+}(z f)\right) * 1
$$

where $\mu_{i}(z f)$ denotes the number of critical points of $z f$ of index $i$ in $M \backslash \partial M$, and $\mu_{i}^{+}(z f)$ denotes the number of $(+)$-critical points of $z f$ of index $i$ in $\partial M$. Here a point $p \in \partial M$ is called $(+)$-critical if $p$ is critical 
for $z f_{\partial M}$ and $\operatorname{grad}_{p} f$ is a nonvanishing inner vector on $M$ (for details, see [2], [4] or [6] ).

The i-th curvature $\tau_{i}$ introduced by N.H. Kuiper (cf. [7] ) can be expressed by

$$
\tau_{i}(f)=\frac{1}{c_{n+k-1}} \quad \int_{z \in S^{n+k-1}}\left(\mu_{i}(z f)+\mu_{i}^{+}(z f)\right) * 1
$$

(cf. [6], lemma 4.2 or [9], lemma 3.1). So we get

$$
T A(f)=\sum_{i} \tau_{i}(f) \text {. }
$$

The Morse-relations give the following connections between the curvatures and some topological invariants of $\mathrm{M}$ :

$$
\begin{aligned}
\tau_{i}(f) & \geq b_{i}(M) \\
T A(f) & \geq b(\mathrm{~m}):=\sum_{i} b_{i}(M) \\
\sum_{i}(-1)^{i} \tau_{i}(f)=x(M) & =\sum_{i}(-1)^{i} b_{i}(M)
\end{aligned}
$$

where $b_{i}(M)$ denotes the $i-t h$ Betti-number of homology with coefficients in a suitable field. (cf. [7]).

$f$ is called $\underline{k \text {-tight }}$ if for all $k^{\prime} \leq k$ and for almost all $z \in s^{n+k-1}$ and all real numbers $c$ the inclusion map

$$
j:(z f)_{c}:=\{p \in M /(z f)(p) \leqq c\} \longrightarrow M
$$

induces a monomorphism in the $\mathrm{k}^{\prime}$-th homology :

$$
\mathrm{H}_{k^{\prime}}(\mathrm{j}): \mathrm{H}_{k^{\prime}}\left((\mathrm{zf})_{\mathrm{c}}\right) \longrightarrow \mathrm{H}_{\mathrm{k}^{\prime}}(\mathrm{M})
$$

As usual we write shortly 'tight' instead of 'n-tight'.

Then the results of N.H. Kuiper show

$$
T A(f)=b(M) \quad \text { if and only if } f \text { is tight, }
$$




$$
\tau_{k}(f)=b_{k}(M) \quad \text { if and only if } \quad H_{k}(j) \text { and } H_{k-1}(j) \text { are monomorphisms }
$$

for almost all z, all c (cf. [7] ).

Results on tightness are collected in the survey article [10] by T.J. Willmore, for results on k-tightness we refer in addition to the notes [1] by $T$. Banchoff and [9] by L. Rodriguez, who has shown that in some sense (n-2)-tightness is closely related to convexity.

\section{RESULTS}

As mentioned above there is a relation between tightness on one hand and total absolute curvature and the sum of the Betti-numbers on the other hand. The following results give certain connections between (n-2)-tightness on one hand and usual curvature terms and the sum of the Betti-numbers on the other hand. Note that in case $\partial M=\phi$ by duality arguments tightness is equivalent to $k$-tightness for $k=\frac{n}{2}-1$ if $n$ is even and for $k=\frac{n-1}{2}$ if $n$ is odd. But in case $\partial M \neq \phi$ there are examples of ( $n-2)$-tight immersions which are not tight (for example: consider the round hemi-sphere).

THEOREM A . Let $M^{\mathrm{n}}$ be an even-dimensional manifold with non-void boundary and $f: M \rightarrow E^{n+k}$ be an immersion. Let $N_{0}$ be the unit normal bundle of $f_{M \backslash a M}$ and denote by $N_{*} \subseteq N_{0}$ the open set of unit normals where the second fundamental form of $f$ is positive or negative definite.

Then there holds the following inequality

$$
\frac{1}{2} \mathrm{TA}\left(\left.\mathrm{f}\right|_{\partial \mathrm{M}}\right)+\frac{1}{\mathrm{c}_{\mathrm{n}+\mathrm{k}-1}} \int_{\mathrm{N}_{0} \backslash \mathrm{N}_{*}}|\mathrm{~K}| *_{1} \geq \mathrm{b}(\mathrm{m})
$$

where equality characterizes (n-2)-tightness of $f$.

In case of hypersurfaces $(k=1)(2.1)$ becomes

$$
\frac{1}{2} T A\left(\left.f\right|_{\partial M}\right)+T A\left(\left.f\right|_{M \backslash M_{*} \backslash \partial M}\right) \geq b(M)
$$


where $M_{*}$ denotes the set of points in $M$ lOM with positive or negative definite second fundamental form.

In case $n=2 M_{*}$ is just the set of points with positive Gaussian curvature, so we get

COROLLARY A 1. Assume $\mathrm{n}=2$ and $\mathrm{k}=1 \cdots$ Then there holds the following inequality

$$
\frac{1}{2 \pi} \int_{K<0}|K| \text { do }+\frac{1}{2 \pi} \int_{\partial M}|x| \text { ds } \geqq b(M) \geq 2-\chi(M)
$$

where equality characterizes 0-tightness of $f$. Here $|\boldsymbol{x}|$ denotes the usual curvature of $f_{\partial M}$ considered as a space curve. For part of this result see [8], Prop. 9.

COROLLARY A 2. Assume $b(\partial M)=2 b(M)$. Then $(n-2)$-tightness of $f$ implies that $\mathrm{f}_{\partial \mathrm{M}}$ is tight and that the second fundamental form of $f$ has either nonmaximal rank of is positive or negative definite.

This is shown in [9], Prop. 5.2 under the assumption that $\mathrm{M}^{\mathrm{n}}$ can be embedded in $E^{n}$. This condition implies $b(\partial M)=2 b(M)$ by Alexander duality. Under the additional assumption that $\partial M$ consists of a certain number of (n-1)-spheres L. Rodriguez has shown that (n-1)-tightness is equivalent to convexity (cf. [9], Theorem 2). This is not true in general, (See Corollary B 2 below).

THEOREM B. Let $n$ be even and $f: M^{n} \rightarrow E^{n+1}$ be $(n-2)-t i g h t$ (if $\partial M \neq \phi$ ) or tight (if $\partial M=\phi$ ), and let $\tilde{M} \leqq M \backslash \partial M$ be a compact submanifold of dimension $n$ which is contained in some coordinate neighborhood in $M$. As above $M_{\star}$ denotes the set of points in $M \backslash \partial M$ with positive or negative definite second fundamental form. Then there holds the following inequality 


$$
\operatorname{TA}(f \mid \tilde{M}) \geq b(\partial \tilde{M})+2 \operatorname{TA}\left(f_{M \backslash M_{\star} \backslash \partial \tilde{M}}\right)
$$

where equality characterizes (n-2)-tightness of $f \mid M(\tilde{M} \backslash \tilde{M})$.

REMARK. If $\tilde{\mathrm{M}}$ contains only points of vanishing curvature or definite second fundamental form, then $\tilde{\mathrm{M}} \mathrm{M}_{\star}=\phi$ and $(2.4)$ reduces to the inequality of S.S Chern and R.K. Lashof for $\partial \tilde{M}$, otherwise (2.4) is sharper and reflects the additional condition that $\tilde{M}$ lies inside of some given $M$. For example in case $\mathrm{n}=2$ and $\tilde{\mathrm{M}}$ being a disk we get

$$
\int_{\partial M}|x| d s \geq 2 \pi+\int_{\tilde{M} \cap\{K<0\}}|K| \text { do }
$$

COROLLARY $B$ 1. Let $f$ be as in Theorem $B$ and assume moreover that there is an open region $U \leqq M$ which is embedded by $f$ in a hyperplane of $E^{n+1}$ which implies $\left.\mathrm{K}\right|_{\mathrm{U}}=0$. Let $\tilde{M}^{\mathrm{n}}$ be an embedded compact submanifold of $\mathrm{E}^{\mathrm{n}}$ and assume by changing the scale $\tilde{\mathrm{M}} \leqq \mathrm{f}(\mathrm{U})$.

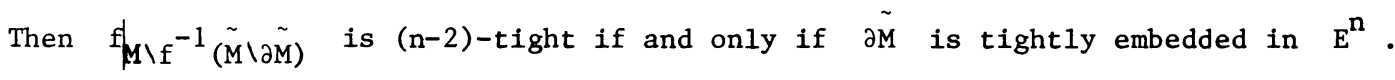
Note that for $\tilde{M}^{n} \leqq E^{n}$ tightness of $\tilde{M}$ and tightness of $\partial \tilde{M}$ are equivalent: this can be obtained easily using the equations $\mathrm{TA}(\tilde{M})=\frac{1}{2} \mathrm{TA}(\partial \tilde{\mathrm{M}})$ and $\mathrm{b}(\tilde{\mathrm{M}})=\frac{1}{2} \mathrm{~b}(\tilde{\partial \mathrm{M}})$.

Roughly spoken Corollary B 1 says: (n-2)-tight minus tight gives (n-2)-tight. In particular we get the following

COROLLARY B 2. In each even dimension there exist (n-2)-tight hypersurfaces which are not tight and not convex in the sense of [9], in particular where $f(\partial M)$ is not contained in the boundary of the convex hull of $f(M)$.

\section{PROOFS.}

In all proofs the immersion $f$ is fixed and so we may write $T A(\partial M)$ instead 
of $T A\left(\left.f\right|_{g M}\right)$ and so on.

PROOF OF THEOREM A.

From

$$
T A(M)=\sum_{i} \tau_{i}(M)
$$

and

we get

$$
\begin{aligned}
\chi(M) & =\sum_{i}(-1){ }^{i} \tau_{i}(M) \\
T A(M)+\chi(M) & =2 \underset{i}{\sum} \tau_{2 i}(M)
\end{aligned}
$$

On the other hand by definition $\tau_{n}(M)$ is the average of the number of critical points of $\mathrm{zf}$ of index $\mathrm{n}$ which are precisely the strict local maxima in $M \backslash \partial M$. But a point is a strict local extremum of some height function $z f$ if and only if the second fundamental form in the direction of $z$ is positive or negative definite. Hence we get

leading to

$$
2 \tau_{n}(M)=\frac{1}{c_{n+k-1}} \int_{N_{\star}}|K| * 1
$$

$$
\begin{aligned}
\operatorname{TA}(M) & -\frac{1}{c_{n+k-1}} \int_{N_{\star}}|K| * 1 \\
& =2\left(\tau_{0}(M)+\tau_{2}(M)+\ldots+\tau_{n-2}(M)\right)-\chi(M) \\
& \geq 2\left(b_{0}(M)+b_{2}(M)+\ldots+b_{n-2}(M)\right)-x(M) \\
& =b(M),
\end{aligned}
$$

where we have used the assumption that $n$ is even and $\partial M \neq \phi$ which implies $b_{n}(M)=0$.

The case of equality is equivalent to the following equations: 


$$
\tau_{0}(M)=b_{0}(M), \tau_{2}(M)=b_{2}(M), \ldots, \tau_{n-2}(M)=b_{n-2}(M)
$$

But the equality $\tau_{i}(M)=b_{i}(M)$ is equivalent to injectivity of $H_{i}(j)$ and $H_{i-1}(j)$ for all inclusions $j:(z f)_{C} \rightarrow M$, so $(2.6)$ is equivalent to (n-2)-tightness of $f$.

The assertion of the theorem then follows from the inequality above using the equation (1.1)

$$
\mathrm{TA}(\mathrm{M})=\mathrm{TA}(\mathrm{M} \backslash \partial \mathrm{M})+\frac{1}{2} \mathrm{TA}(\partial \mathrm{M})
$$

PROOF of Corollary A 2. By theorem A (n-2)-tightness of $f$ implies

$$
\begin{aligned}
b(M) & =\frac{1}{2} \mathrm{TA}(\partial \mathrm{M})+\frac{1}{c_{n+k-1}} \int_{N_{0} \backslash N_{*}}|K| * 1 \\
& \geqq \frac{1}{2} \mathrm{TA}(\partial \mathrm{M}) \geqq \frac{1}{2} \mathrm{~b}(\partial \mathrm{M})=b(\mathrm{M})
\end{aligned}
$$

which implies tightness of $\left.f\right|_{\partial M}$ and moreover the vanishing of the integral of $|K|$ over $N_{O} \backslash N_{*}$, hence $K=0$ on $N_{O} \backslash N_{*}$.

PROOF of Theorem B. By assumption and by theorem A we have

$$
\begin{aligned}
\mathrm{TA}_{\left(M \backslash M_{*} \backslash \partial \mathrm{M}\right)+\frac{1}{2} \mathrm{TA}(\partial \mathrm{M})} & =\mathrm{b}(\mathrm{M}), \text { if } \partial \mathrm{M} \neq \phi, \\
\text { or } & \mathrm{TA}(\mathrm{M})=\mathrm{b}(\mathrm{M}), \text { if } \partial \mathrm{M}=\phi
\end{aligned}
$$

which last equality is equivalent to

$$
\mathrm{TA}\left(\mathrm{M} \backslash \mathrm{M}_{*}\right)=\mathrm{b}(\mathrm{M})-2
$$

For $f_{M \backslash(\tilde{M} \backslash \partial \tilde{M})}$ theorem A yields

$$
\mathrm{TA}\left(\mathrm{M} \backslash \tilde{\mathrm{M}} \backslash \mathrm{M}_{\star} \backslash \partial \mathrm{M} \backslash \partial \tilde{\mathrm{M}}\right)+\frac{1}{2} \mathrm{TA}(\partial \mathrm{M})+\frac{1}{2} \mathrm{TA}(\partial \tilde{\mathrm{M}}) \geqq b(\mathrm{M} \backslash \tilde{\mathrm{M}})
$$

where equality characterizes $(n-2)$-tightness of $\left.f\right|_{M \backslash(\tilde{M} \backslash \partial \tilde{M})}$. Subtracting (2.9) from (2.7) or (2.8) respectively we get 


$$
\begin{aligned}
\operatorname{TA}\left(\tilde{M} \backslash M_{\star} \backslash \tilde{\partial M}\right)-\frac{1}{2} \mathrm{TA}(\tilde{\partial M}) & \leqq b(M)-b(M \backslash \tilde{M}) \\
\tilde{T A}\left(\tilde{M} \backslash M_{*} \backslash \tilde{\partial M}\right)-\frac{1}{2} \operatorname{TA}(\tilde{\partial M}) & \leqq b(M)-b(M \backslash \tilde{M})-2
\end{aligned}
$$

respectively.

Now the assertion follows directly from the following lemma

LEMMA. Let $M, \tilde{M}$ be n-dimensional dompact connected manifolds with $\tilde{M} \leqq M I \partial M$ and assume that $\tilde{M}$ is contained in some coordinate neighborhood of $M$ Then

$$
\begin{aligned}
& b(M) \tilde{M})-b(M)=\frac{1}{2} b(\partial \tilde{M}) \text { if } \partial M \neq \phi, \\
& \text { or } \quad b(\tilde{M} \backslash \tilde{M})-b(M)=\frac{1}{2} b(\partial \tilde{M})-2 \text { if } \partial M=\phi
\end{aligned}
$$

PROOF. Let $B$ be an open coordinate neighborhood in $M$ such that $\bar{B}$ is topologically a closed n-ball. We can assume $\tilde{\mathrm{M}} \leqq \mathrm{B} \leqq \overline{\mathrm{B}} \leqq \mathrm{M} \backslash \partial \mathrm{M}$. To compute the Betti-numbers of $\tilde{M} \tilde{M}$ in terms of that of $M$ and $\tilde{M}$ we apply the MayerVietoris sequence to the following three decompostions

I.

$$
\begin{aligned}
M= & (M \backslash B) \cup \bar{B} \\
& (M \backslash B) \cap \bar{B}=\partial \bar{B} \cong S^{n-1},
\end{aligned}
$$

II.

$$
\bar{B}=(\bar{B} \backslash \tilde{M} \backslash \partial \tilde{M})) \cup \tilde{M}
$$$$
(\bar{B} \backslash \tilde{(M} \backslash \tilde{M})) \cap \tilde{M}=\tilde{M} \text {, }
$$

III.

$$
\begin{aligned}
M \backslash \tilde{M} \backslash \tilde{M})= & (M \backslash B) \cup(\bar{B} \backslash \tilde{M} \backslash \tilde{M})) \\
& (M \backslash B) \cap(\bar{B} \backslash \tilde{M} \backslash \tilde{M}))=\partial \bar{B} \cong \mathrm{S}^{n-1} .
\end{aligned}
$$

The first decomposition leads to

$$
\begin{array}{ll}
b(M)=b(M \backslash B)-1 & \text { if } \partial M \neq \phi \\
b(M)=b(M \backslash B)+1 & \text { if } \partial M=\phi,
\end{array}
$$


the second one to

$$
b(B \backslash \tilde{M})+b(\tilde{M})=b(\partial \tilde{M})+1
$$

the third one to

$$
b(\tilde{M} \tilde{M})=b(M \backslash B)+b(\bar{B} \backslash \tilde{M})-2
$$

At last we have the equation

$$
\mathrm{b}(\partial \tilde{M})=2 \mathrm{~b}(\tilde{M})
$$

because by assumption $\tilde{M}$ can be embedded in $B \leqq E^{n}$ (cf. [9] Prop. 5.1).

Now the lemma follows directly from (2.12) - (2.16) .

PROOF of Corollary B 2 . Consider for example an embedding of $S^{k} \times S^{n-k}$ in $E^{n+1} \quad(k \geq 1$ arbitrary) as a tight hypersurface of rotation (like the standard-torus in $\mathrm{E}^{3}$ ) and change this embedding a little bit such that there is an open region $U$ contained in some hyperplane of $E^{n+1}$. Now define $M$ by removing a small tight 'solid torus' of type $\mathrm{S}^{\mathrm{m}} \times \mathrm{B}^{\mathrm{n}-\mathrm{m}}$ from $\mathrm{U}(\mathrm{m} \gg 1)$. By Corollary B $1 \mathrm{M}$ is (n-2)-tight but of course it is not tight. By suitable choice of the embedding of $S^{k} \times S^{n-k}$ we started from we can assume that $U$ lies not in the boundary of the convex hull $X M$. So we can obtain an example were $\partial M$ lies not in the boundary of $M$.

REMARK. In the examples of corollary B 2 the boundary $O M$ was always tightly embedded in $\mathbf{E}^{\mathrm{n}+1}$. The natural question whether there exist in higher dimensions (n-2)-tight immersions with non-tight boundary seems to be open. For $n=2$ an example is due to L. Rodriguez. 


\section{REFERENCES}

1. Banchoff, T.F. The two-piece-property and tight n-manifolds-with-boundary in $\mathrm{E}^{\mathrm{n}}$, Trans. Amer. Math. Soc. 161 (1971) 259-267.

2. Braess, D. Morse-Theorie für berandete Mannigfaltigkeiten, Math. Ann. 208 (1974) 133-148.

3. Chern, S.S. and R.K. Lashof, On the total curvature of immersed manifolds, I, II, Amer. J. Math. 79 (1957) 306-313, Mich. Math. J. 5 (1958) 5-12.

4. Friedrich, T. m-Funktionen und ihre Anwendung auf die totale Absolutkrümung, Math. Nachr. 67 (1975) 281-301.

5. Grossman, N. Relative Chern-Lashof theorems, J. Diff. Geom. 7 (1972) 607-614.

6. Kühnel, W. Total curvature of manifolds with boundary in $E^{n}$, London Math Soc. (2) 15 (1977) 173-182.

7. Kuiper, N.H. Morse relations for curvature and tightness, in: Proc. Liverpool Singularities Symp. II (ed.: C.T.C. Wal1), 77-89, Springer 1971 (Lecture Notes in Mathematics 209).

8. Rodriguez, L.L. The two-piece-property for surfaces with boundary, J. Diff. Geom. 11 (1976) 235-250.

9. Rodriquez, L. L. Convexity and tightness of manifolds with boundary, in: Geometry and Topology, Proc. of III Latin American School of Mathematics (ed1: J. Palis and M. Do Carmo), 510-541, Springer 1977 (Lecture Notes in Mathematics 597).

10. Willmore, T. J. Tight immersions and total absolute curvature, Bu11. London Math.Soc. 3 (1971) 129-151. 


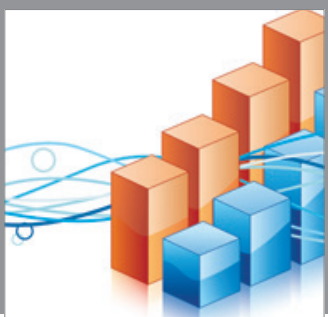

Advances in

Operations Research

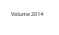

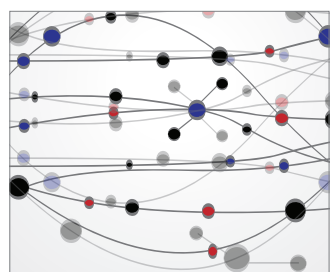

\section{The Scientific} World Journal
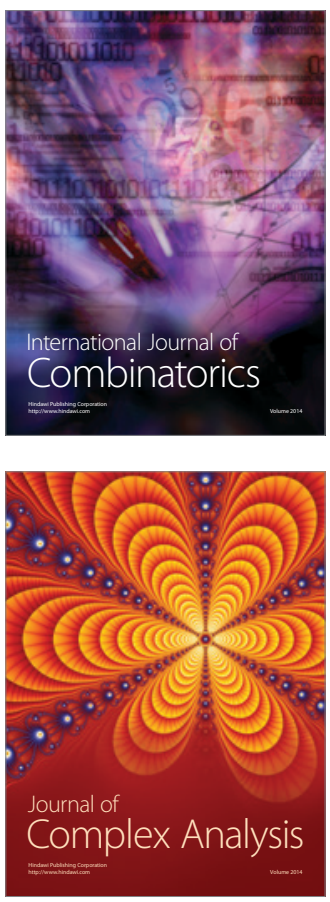

International Journal of

Mathematics and

Mathematical

Sciences
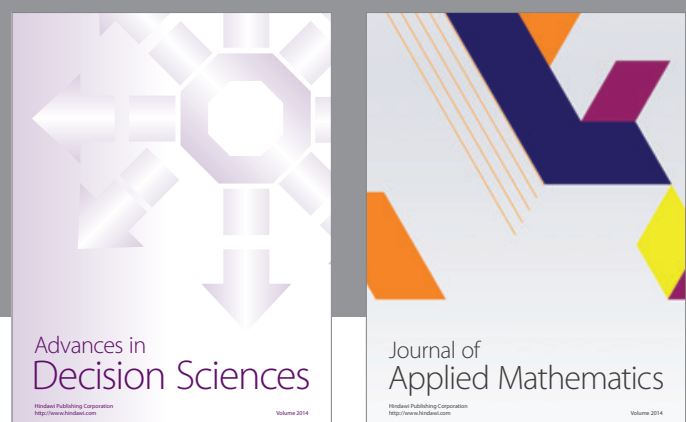

Journal of

Applied Mathematics
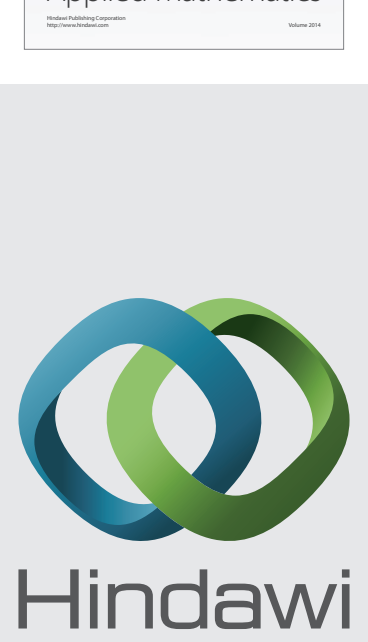

Submit your manuscripts at http://www.hindawi.com
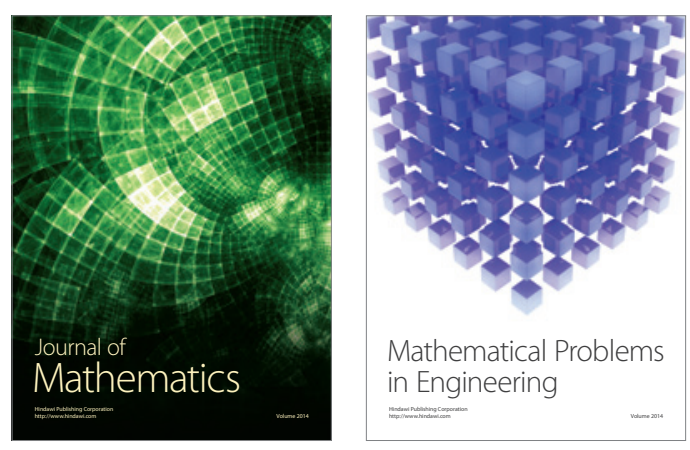

Mathematical Problems in Engineering
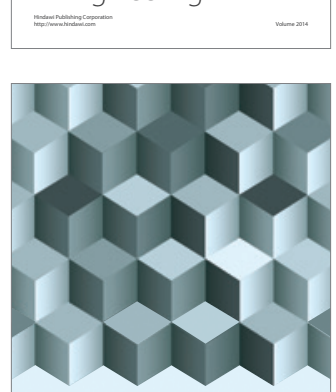

Journal of

Function Spaces
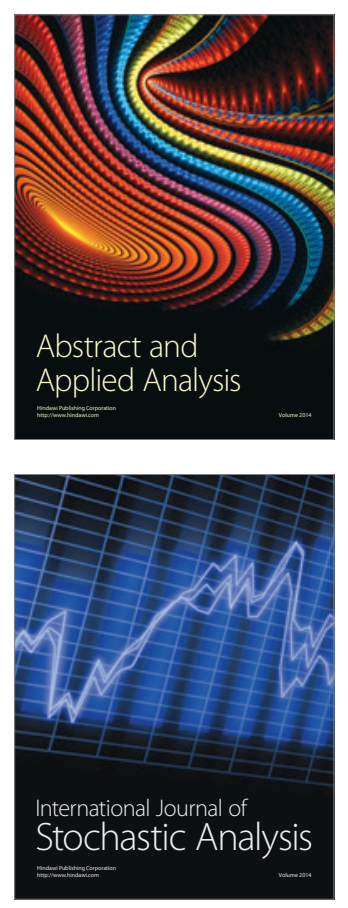

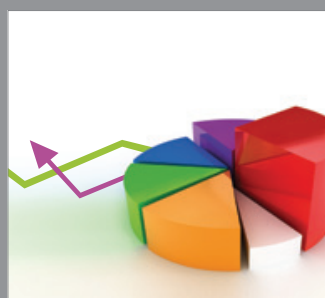

ournal of

Probability and Statistics

Promensencen
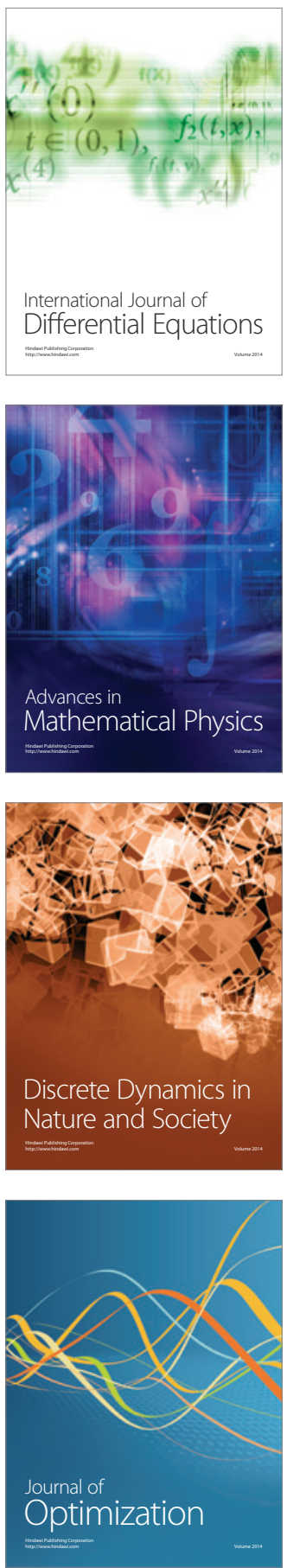